T. Matsuzawa

Nagoya Math. J.

Vol. 66 (1977), 1-12

\title{
HYPOELLIPTICITY FOR A CLASS OF THE SECOND ORDER PARTIAL DIFFERENTIAL EQUATIONS
}

\author{
TADATO MATSUZAWA
}

\section{\$1. Introduction.}

In this paper, we shall investigate the hypoellipticity for a class of degenerate equations of the second order with complex coefficients as a direct extension of the results obtained in [8]. As is well known, the satisfactory general results about hypoellipticity of real operators of the second order have been obtained in [3] and [9], where the assumption that the operators are real plays a crucial role and our aim of this paper is to study the operators with complex coefficients. Our method may be considered as a generalization of the usual variational method replacing the Gårding inequality by the estimate (2.15), (cf. [3], [5]).

Let $\boldsymbol{R}^{N}$ be $N$-dimensional Euclidean space regarded as a direct product of three Euclidean spaces $\boldsymbol{R}_{x}^{m}, \boldsymbol{R}_{y}^{n}$ and $\boldsymbol{R}_{t}^{1}(m+n+1=N)$ and generic point of $\boldsymbol{R}^{N}$ will be denoted by $(x, y, t)=\left(x_{1}, \cdots, x_{m}, y_{1}, \cdots, y_{n}, t\right)$. We shall mainly consider a partial differential equation of the form

$$
\begin{aligned}
L(x, y, t, D)= & D_{t} u-\sum_{k, j=1}^{m} D_{x_{j}}\left(a^{k i} D_{x_{k}} u\right)-\sum_{k, j=1}^{m} D_{y_{j}}\left(a_{1}^{k j} D_{y_{k}} u\right) \\
& -2 \sum_{k=1}^{m} \sum_{j=1}^{n} D_{y_{j}}\left(g^{k j} D_{x_{k}} u\right)+\sum_{k=1}^{m} b^{k} D_{x_{k}} u+\sum_{j=1}^{n} b_{1}^{j} D_{y_{j}} u \\
& +c u=f \quad \text { in } \Omega,
\end{aligned}
$$

where $D_{x_{j}}=\partial / \partial x_{j}$ and $a^{k j}, a_{1}^{k j}, g^{k j}, b^{k}, b_{1}^{j}, c$ and $f$ are complex valued $C^{\infty}$ functions defined in a domain $\Omega \subset \boldsymbol{R}^{N}$ which is supposed to contain the origin $\{0\}$ of $\boldsymbol{R}^{N}$.

The following notations are convenient for the later discussions:

$$
\begin{aligned}
& A=\left(\alpha^{k j}(x, y, t)\right)_{1 \leqq k, j \leqq m}, \quad \mathscr{R}_{e} A=\left(\mathscr{R} e a^{k j}\right)_{1 \leqq k, j \leqq m}, \\
& \operatorname{Im} A=\left(\operatorname{Im} \alpha^{k j}\right)_{1 \leqq k, j \leqq m},
\end{aligned}
$$

Received September 18, 1975. 


$$
\begin{aligned}
A_{x_{\sigma}} & =\left(a_{x_{\sigma}}^{k j}(x, y, t)\right)_{1 \leqq k, j \leqq m}, \\
Q_{0} & =Q_{0}(x, y, t, D)=D_{t}+\sum_{k=1}^{m} \mathscr{R} e b^{k} D_{x_{k}}, \\
Q_{j} & =\sum_{k=1}^{m} \mathscr{R} e a^{k j} D_{x_{k}}, j=1, \cdots, m, \quad Q_{m+j}=D_{y_{j}}, j=1, \cdots, n .
\end{aligned}
$$

Now we set the hypotheses on $L$ :

$$
\begin{gathered}
\mathscr{R} e \sum_{k, j=1}^{m} a^{k j} \xi_{k} \xi_{j} \geqq 0 \quad \text { in } \Omega \text { for all } \xi \in \boldsymbol{R}^{m}, \\
a^{k k}(0)=0, k=1, \cdots, m, \quad a^{k j}=a^{j k}, \quad 1 \leqq j, k \leqq m, \\
\mathscr{R} e \sum_{k, j=1}^{n} a_{1}^{k j} \eta_{k} \eta_{j} \geqq \alpha|\eta|^{2} \quad \text { in } \Omega, \eta \in \boldsymbol{R}^{n}(\alpha>0) . \\
\sum_{\sigma=1}^{m}\left[\mathscr{R}_{e} A_{x_{\sigma}}\right]^{2}+\left[\mathscr{R}_{e} A_{t}\right]^{2} \leqq C^{1)} \mathscr{R}_{e} A \quad \text { in } \Omega, \\
\left|\operatorname{Im} a^{k j} \sum_{k, j=1}^{m} \xi_{k} \xi_{j}\right| \leqq C \mathscr{R} e \sum_{k, j=1}^{m} a^{k j} \xi_{k} \xi_{j} \quad \text { in } \Omega, \xi \in \boldsymbol{R}^{m},
\end{gathered}
$$

(1.7) every vector field $D_{x_{j}}, j=1, \cdots, m$, (on $\Omega$ ) can be expressed as a linear combination (with $C^{\infty}$ coefficients) of $Q_{0}, Q_{1}, \cdots, Q_{m+n}, \cdots$, $\left[Q_{k}, Q_{j}\right], \cdots,\left[Q_{i},\left[Q_{k}, Q_{j}\right]\right], \cdots,\left[Q_{j_{1}},\left[Q_{j_{2}}, \cdots, Q_{j_{p}}\right], \cdots,\right], \cdots$,

(1.8) denoting by $A_{1}=\left(a_{1}^{k j}\right)_{1 \leqq k, j \leqq n}$ and $G=\left(g^{k j}\right)_{1 \leqq k \leqq m, 1 \leqq j \leqq n}$, we have

$$
\mathscr{R e}\left(\begin{array}{cc}
\mu A & G \\
{ }^{t} G & \mu A_{1}
\end{array}\right) \geqq 0 \quad \text { in } \Omega
$$

for some positive constant $\mu, 0<\mu<1$,

$$
\begin{array}{cc}
\sum_{j=1}^{n}\left|\sum_{k=1}^{m} g^{k j} \xi_{k}\right|^{2} \leqq C \mathscr{R} e a^{k j} \xi_{k} \xi_{j} & \text { in } \Omega, \xi \in \boldsymbol{R}^{m}, \\
\left|\sum_{k=1}^{m} \operatorname{Im} b^{k} \xi_{k}\right|^{2} \leqq C \mathscr{R} e a^{k j} \xi_{k} \xi_{j} & \text { in } \Omega, \xi \in \boldsymbol{R}^{m} .
\end{array}
$$

We remark that there is no restriction on $\mathscr{R} e b^{k}, k=1, \cdots, m$.

Our main result is to prove the following theorem.

THEOREM 1.1. Suppose that the operator $L$ given in (1.1) satisfies the condition $(1.2) \sim(1.10)$. Then any distribution $u \in \mathscr{D}^{\prime}(\Omega)$ satisfying (1.1) with $f \in C^{\infty}(\Omega)$ must be a $C^{\infty}$ function in $\Omega$.

EXAMPLES. The following operators satisfy the above conditions:

1) We use the symbols $C, C^{1}, \cdots$ to express the different positive constants throughout this paper. 
1) $L=D_{t}-a t^{2}\left(t^{2}+y^{2}\right) D_{y}^{2}$ in $\boldsymbol{R}^{2}, \mathscr{R}_{e} a>0$,

2) $L=D_{t}-y^{2}\left(x^{2}+y^{2}\right) D_{x}^{2}-D_{y}^{2}+(1+i a) x y D_{x} D_{y}+b D_{x}$ in $R_{x, y, t}^{3}, a, b$ real,

3) degenerate elliptic operator treated in [8] considered as a stationary case in the variable $t$ in (1.1).

The inequality (2.10) plays an essential role in the proof of Theorem 1.1 and the hypothese (1.7) is a sufficient condition so that (2.10) is valid. We can get the following result by the same manner as in Theorem 1.1:

Let $\boldsymbol{R}^{N}$ be $N$-dimensional Euclidean space regarded as a direct product of three Euclidean spaces $\boldsymbol{R}^{m}, \boldsymbol{R}^{n}$ and $\boldsymbol{R}^{p}(m+n+p=N)$ and generic point of $\boldsymbol{R}^{N}$ will be denoted by $(x, y, t)=\left(x_{1}, \cdots, x_{m}, y_{1}, \cdots, y_{n}\right.$, $\left.t_{1}, \cdots, t_{p}\right)$.

We consider a partial differential equation of the form

$$
\begin{aligned}
L(x, y, t, D) u= & -\sum_{k, j=1}^{m} D_{x_{j}}\left(a^{k j} D_{x_{k}} u\right)-\sum_{k, j=1}^{m} D_{y_{j}}\left(a_{1}^{k j} D_{y_{k}} u\right) \\
& -2 \sum_{k=1}^{m} \sum_{j=1}^{n} D_{y_{j}}\left(g^{k j} D_{x_{k}} u\right)+\sum_{k=1}^{m} b^{k} D_{x_{k}} u \\
& +\sum_{j=1}^{n} b_{1}^{j} D_{y_{j}} u+\sum_{\ell=1}^{p} d^{\ell} D_{t_{\ell}} u+c u=f \quad \text { in } \Omega,
\end{aligned}
$$

where $a^{k j}, a_{1}^{k j}, g^{k j}, b^{k}, b_{1}^{j}, c$ and $f$ are complex valued $C^{\infty}$ functions in $\Omega \subset$ $R^{N}$ as in (1.1) and we remark that only the coefficients $d^{\ell}=d^{\ell}(x, y, t)$ $(\ell=1, \cdots, p)$ are supposed to be real valued $C^{\infty}$ functions in $\Omega$.

THEOREM 1.2. For the operator $L$ defined by $(1.1)^{\prime}$ we suppose that the hypotheses $(1.2) \sim(1.6),(1.8) \sim(1.10)$ and the estimate $(2.10)$ are valid, where we take

$$
Q_{0}=\sum_{k=1}^{m} \mathscr{R} e b^{k} \frac{\partial}{\partial x_{k}}+\sum_{\ell=1}^{p} d^{\ell} \frac{\partial}{\partial t_{\ell}}
$$

and

$$
\sum_{1 \sigma=}^{m}\left[\mathscr{R}_{e} A_{x_{\sigma}}\right]^{2}+\sum_{\ell=1}^{p}\left[\mathscr{R}_{e} A_{t_{\ell}}\right]^{2} \leqq C \mathscr{R e} A \quad \text { in } \Omega
$$

Then any distribution $u \in \mathfrak{D}^{\prime}(\Omega)$ satisfying $(1.1)^{\prime}$ with $f \in C^{\infty}(\Omega)$ must be a $C^{\infty}$ function in $\Omega$.

EXAMPLE. The following operator satisfies the condition of Theorem 1.2: 
4) $L=a_{1} D_{y}^{2}+d y^{k} D_{t}$,

$\mathscr{R}_{e} a_{1}>0, k$ integer $\geqq 0$,

$d$ real, $d \neq 0$ : Fokker-Plank type.

Finally, we remark that we can prove the hypoellipticity of the first boundary value problem for the equation (1.1) by the similar way as in [8].

\section{§2. Preliminaries for the proof of Theorem 1.1 .}

The proof will be obtained by the same steps as in the proof of Theorem 1.1 of [8]. Suggested by [8], we introduce the norm ||$|\cdot| \mid$ and its dual norm $\||\cdot|\|^{\prime}$ by

$$
\begin{aligned}
& \left.\|u\|\right|^{2}=\sum_{k, j=1}^{m} \int_{\Omega} \mathscr{R}_{e} a^{k i} u_{x_{j}} \bar{u}_{x_{j}} d x d y d t+\sum_{j=1}^{n}\left\|u_{y_{j}}\right\|^{2}+\|u\|^{2}, \\
& \|v\| \|^{\prime}=\sup _{w \in C_{0}^{\infty}(\Omega)} \frac{|\langle v, w\rangle|}{\|w\|},
\end{aligned}
$$

where $\|\cdot\|$ is the usual $L^{2}$-norm on $\Omega$ and $\langle v, w\rangle$ is the value of $v \in \mathscr{D}^{\prime}(\Omega)$ evaluated at $w$.

LEMMA 2.1. Let $L$ be the operator given in (1.1). We have the following estimate with some positive constant $C$ :

$$
\|v\|\|+\| Q_{0} v \|\left.\right|^{\prime} \leqq C\left(\|v\|+\left.\|L v\|\right|^{\prime}\right), \quad v \in C_{0}^{\infty}(\Omega) .
$$

Proof. Obviously we have

$$
|\langle L v, \bar{v}\rangle| \leqq|| L v\left|\left\|^{\prime}|| v \mid\right\|, \quad v \in C_{0}^{\infty}(\Omega) .\right.
$$

Next, integrating by parts, we have

$$
\begin{aligned}
\mathscr{R} e\langle L v, \bar{v}\rangle= & \sum_{k, j=1}^{m} \int_{\Omega}\left(\mathscr{R} e a^{k j}\right) v_{x_{k}} \bar{v}_{x_{j}} d V+\sum_{k, j=1}^{n} \int_{\Omega}\left(\mathscr{R}_{e} a_{1}^{k j}\right) v_{y_{k}} \bar{v}_{y_{j}} d V \\
& +2 \sum_{k=1}^{m} \sum_{j=1}^{n} \int_{\Omega}\left(\mathscr{R} e g^{k j}\right) v_{x_{k}} \bar{v}_{y_{j}} d V \\
& +\mathscr{R} e \int_{\Omega} Q_{0} v \cdot \bar{v} d V-\operatorname{Im} \sum_{k=1}^{m} \int_{\left(\operatorname{Im} b^{k}\right) v_{x_{k}} \bar{v} d V} \\
& +\mathscr{R} e \sum_{j=1}^{n} \int_{\Omega} b_{1}^{j} v_{y_{j}} \bar{v} d V+\mathscr{R} e \int_{\Omega} C|v|^{2} d V,
\end{aligned}
$$

where $d V=d x d y d t=d x_{1} \cdots d x_{m} d y_{1} \cdots d y_{n} d t$. By virtue of the hypotheses (1.4), (1.8) and (1.9) we have easily

$$
\mathscr{R} e\langle L v, \bar{v}\rangle \geqq C_{1}\|v\|\left\|^{2}-C_{2}\right\| v \|^{2}, \quad v \in C_{0}^{\infty}(\Omega)
$$


for some positive constants $C_{1}$ and $C_{2}$. With a new constant $C$ we obtain from (2.2) and (2.3)

$$
\mid\|v\| \| \leqq C\left(\|v\|+\|L v\| \|^{\prime}\right), \quad v \in C_{0}^{\infty}(\Omega) .
$$

Noting that

$$
\begin{aligned}
Q_{0} v= & L v+\sum_{k, j=1}^{m}\left(a^{k j} v_{x_{k}}\right)_{x_{j}}+\sum_{k, j=1}^{n}\left(a_{1}^{k j} u_{y_{k}}\right)_{y_{j}} \\
& +2 \sum_{k=1}^{m} \sum_{j=1}^{n}\left(g^{k j} v_{x_{k}}\right)_{y_{j}}-i \sum_{k=1}^{m} \operatorname{Im} b^{k} \cdot v_{x_{k}} \\
& -\sum_{j=1}^{n} b_{1}^{j} v_{y_{j}}-c v,
\end{aligned}
$$

and using the conditions (1.4), (1.6), (1.9) and (1.10) we easily obtain for some constant $C$

$$
\left\|Q_{0} v\right\| \|^{\prime} \leqq C\left(\|L v\|\left\|^{\prime}+\right\| v\|\|\right), \quad v \in C_{0}^{\infty}(\Omega) .
$$

Indeed, for example, we have by (1.4)

$$
\begin{aligned}
\left|\left\langle\sum_{k, j=1}^{m}\left(a^{k j} v_{x_{k}}\right)_{x_{j}}, w\right\rangle\right| & =\left|\sum_{k, j=1}^{m}\left\langle a^{k j} v_{x_{k}}, w_{x_{j}}\right\rangle\right| \\
& \leqq C\||\|v\||\| \cdot \| w|| \mid w \in C_{0}^{\infty}(\Omega),
\end{aligned}
$$

Thus we have

$$
\left\|\sum_{k, j=1}^{m}\left(a^{k j} v_{x_{k}}\right)_{x_{j}}\right\|\left\|^{\prime} \leqq C \mid\right\| v\|\|, \quad v \in C_{0}^{\infty}(\Omega) .
$$

Other terms can be treated similarly. We get the estimate (2.1) combining (2.4) with (2.5).

LEMMA 2.2. Let $L$ be as above, then we have

$$
\begin{gathered}
\sum_{j=1}^{m}\left\|\sum_{k=1}^{m} a_{x_{\sigma}}^{k j} v_{x_{k}}\right\|\left\|^{\prime} \leqq C\right\| v \|, \quad \sigma=1, \cdots, m, \\
\sum_{j=1}^{m}\|\| \sum_{k=1}^{m} a_{t}^{k j} v_{x_{k}}\|\|^{\prime} \leqq C\|v\|, \\
\sum_{j=1}^{m}\|\| \sum_{k=1}^{m} a^{k j} v_{x_{k}}\|\|^{\prime} \leqq C\|v\|, \\
\left\|\sum_{k=1}^{m} \operatorname{Im} b^{k} \cdot v_{x_{k}}\right\|\left\|^{\prime} \leqq C\right\| v \| \\
\sum_{j=1}^{n}\left\|v_{y_{j}}\right\|\left\|^{\prime} \leqq C\right\| v \|
\end{gathered}
$$


for all $v \in C_{0}^{\infty}(\Omega)$ with some positive constant $C$.

Proof. For any $w \in C_{0}^{\infty}(\Omega)$, we have

$$
\left\langle\sum_{k, j=1}^{m} a_{x_{\sigma}}^{k j} v_{x_{k}}, w\right\rangle=-\sum_{j=1}^{m}\left\langle v, \sum_{k=1}^{m} a_{x_{\sigma}}^{k j} w_{x_{k}}\right\rangle-\sum_{j=1}^{m}\left\langle v, \sum_{k=1}^{m} a_{x_{\sigma} x_{k}}^{k j} w\right\rangle \text {. }
$$

Taking account of the assumption (1.5), we have (2.6) by applying Schwartz inequality for the right hand side of the above equality. By the similar way we have $\left(2.6^{\prime}\right)$ and (2.7). Finally for $w \in C_{0}^{\infty}(\Omega)$ we have

$$
\left\langle\sum_{k=1}^{m} \operatorname{Im} b^{k} \cdot v_{x_{k}}, w\right\rangle=-\left\langle v, \sum_{k=1}^{m} \operatorname{Im} b^{k} \cdot w_{x_{k}}\right\rangle-\left\langle v, \sum_{k=1}^{m} \operatorname{Im} b_{x_{k}}^{k} \cdot w\right\rangle,
$$

which gives the estimate (2.8) by assumption (1.10). (2.9) is trivially obtained.

Now we introduce the norm $\|\cdot\|_{(s, r)}$, with $s$ any real number and $r$ nonnegative integer (cf. [2], §2.6), defined by

$$
\begin{aligned}
& \|v\|_{(s, r)}^{2}=(2 \pi)^{-(m+1)} \int_{R_{y}^{n}} \int_{R_{\xi, \tau}^{m+1}}|\hat{V}(\xi, y, \tau)|^{2}\left(1+|\xi|^{2}+|\tau|^{2}\right)^{s} d \xi d y d \tau
\end{aligned}
$$

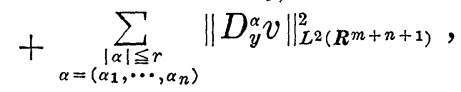

$$
\begin{aligned}
& \xi=\left(\xi_{1}, \cdots, \xi_{m}\right) \in \boldsymbol{R}_{\xi}^{m}, \quad \tau \in \boldsymbol{R}_{\tau}^{1}, \\
& \hat{V}(\xi, y, \tau)=\iint e^{-i\langle\langle x, \hat{\xi}\rangle\langle\langle t, \tau\rangle)} v(x, y, t) d x d t, \quad v \in C_{0}^{\infty}\left(\boldsymbol{R}_{x, y, t}^{m+n+1}\right) .
\end{aligned}
$$

We denote by $H_{(s, r)}\left(\boldsymbol{R}_{x, y, t}^{m+n+1}\right)$ the completion of $C_{0}^{\infty}\left(\boldsymbol{R}^{m+n+1}\right)$ in the norm $\|\cdot\|_{(s, r)}$.

Lemma 2.3. For any compact subset $K$ of $\Omega$, there exist positive constants $\varepsilon$ and $(0<\varepsilon \leqq 1)$ and $C$ such that

$$
\|v\|_{(s, 1)} \leqq C\left(\|v\|\|+\| Q_{0} v \|\left.\right|^{\prime}\right), \quad v \in C_{0}^{\infty}(K) .
$$

Proof. We shall use tentatively the following notation:

$$
\begin{aligned}
\|v\|_{(s)}^{2} & =\int|\hat{v}(\xi, \eta, \tau)|^{2}\left(1+|\xi|^{2}+|\eta|^{2}\right)^{s} d \xi d \eta d \tau, \quad s \text { real } \\
\|v\|_{s}^{2} & =\int|\hat{v}(\xi, \eta, \tau)|^{2}\left(1+|\xi|^{2}+|\eta|^{2}+|\tau|^{2}\right)^{s} d \xi d \eta d \tau .
\end{aligned}
$$

Then by the assumption (1.7), we can apply the results of [3], $\S 4,5$ or the idea of [5] to get the following estimate for some number $\varepsilon^{\prime}, 0<$ $\varepsilon^{\prime} \leqq 1$ : 


$$
\|v\|_{\left({\varepsilon^{\prime}}^{\prime}\right)} \leqq \sum_{j=1}^{m+n}\left\|Q_{j} v\right\|, \quad v \in C_{0}^{\infty}(K) .
$$

On the other hand, clearly we have

$$
\sum_{j=1}^{m+n}\left\|Q_{j} v\right\| \leqq C\|v\| \|, \quad v \in C_{0}^{\infty}(K) .
$$

Since $Q_{0} v=v_{t}+\sum_{k=1}^{m} \mathscr{R} e b^{k} v_{x_{k}}$, it follows that

$$
\left\|D_{t} v\right\|_{(-1)} \leqq C\left(\left\|Q_{0} v\right\|\left\|^{\prime}+\right\| v \|\right) \text {. }
$$

Hence we have

$$
\|v\|_{\left(^{\prime}\right)}+\left\|D_{t} v\right\|_{(-1)} \leqq C\left(\left\|\left|Q_{0} v\left\|\left.\right|^{\prime}+\right\| v \|\right|\right), \quad v \in C_{0}^{\infty}(K),\right.
$$

from which we have

$$
\|v\|_{\varepsilon^{\prime} /\left(1+\varepsilon^{\prime}\right)} \leqq C\left(\|\| Q_{0} v\left\|\left.\right|^{\prime}+\right\| v\|\|\right), \quad v \in C_{0}^{\infty}(K) .
$$

Thus by the definition of norm $\|\cdot \mid\|$ and $Q_{m+j}=D_{y_{j}}, j=1, \cdots, n$, the estimate (2.10) follows with $\varepsilon=\varepsilon^{\prime} /\left(\varepsilon^{\prime}+1\right)$.

Q.E.D.

Combining (2.10) with (2.1) we now come to the main estimate:

$$
\|v\|_{(\varepsilon, 1)} \leqq C\left(\|v\|+\|L v\|^{\prime}\right), \quad v \in C_{0}^{\infty}(K) .
$$

In $\S 3$, we shall prove that it follows from (2.15) that $L$ is hypoelliptic in $\Omega$.

\section{§3. Proof of Theorem 1.1.}

The main step of the proof is to prove the following lemma which corresponds to Proposition 3.1 of [3].

LEMMA 3.1. Every $v \in H_{(0,2)}\left(\boldsymbol{R}^{m+n+1}\right) \cap \mathscr{E}^{\prime}(\Omega)$ such that $\|L v\| \|^{\prime}<\infty$ belongs to $H_{(\varepsilon, 2)}\left(\boldsymbol{R}^{m+n+1}\right) \cap \mathscr{E}^{\prime}(\Omega)$ with a positive number $\varepsilon$, where $\Omega$ is shrinked if necessary.

Proof. We can easily see that (2.11) is valid for all $v \in H_{(2.2)}\left(\boldsymbol{R}^{m+n+1}\right)$ $\cap \mathscr{E}^{\prime}(\Omega)$ as in the proof of Lemma (2.6) of [8]. Next if $v$ satisfies the required conditions, we choose $\chi \in C_{0}^{\infty}(\Omega)$ so that $0 \leqq \chi \leqq 1$ and $\chi=1$ in a neighbourhood $\omega$ of supp. $v$ and we set

$$
v_{\delta}=\chi\left(1-\delta^{2} \Delta\right)^{-1} v, \quad \delta>0, \quad \Delta=\sum_{k=1}^{m} \frac{\partial^{2}}{\partial x_{k}^{2}}+\frac{\partial^{2}}{\partial t^{2}} .
$$

Here $\left(1-\delta^{2} \Delta\right)^{-1} v$ is defined as the Fourier transform of 


$$
\begin{aligned}
& \left(1+\delta^{2}\left(|\xi|^{2}+|\tau|^{2}\right)\right)^{-1} \hat{v}(\xi, y, \tau), \quad \xi=\left(\xi_{1}, \cdots, \xi_{m}\right), \\
& v_{\grave{o}}=\chi \cdot(2 \pi)^{-m-1} \int_{R_{\tilde{\xi},:}^{m+1}} e^{i(\langle x, \hat{\varsigma}\rangle+\langle t, \tau\rangle)}\left(1+\delta^{2}\left(|\xi|^{2}+|\tau|^{2}\right)\right)^{-1} \hat{v}(\xi, y, \tau) d \xi d \tau .
\end{aligned}
$$

It is clear that $v_{\delta}$ is then in $H_{(2)}\left(\boldsymbol{R}^{m+n+1}\right) \cap \mathscr{G}^{\prime}(\Omega)$, and that $v_{\delta} \rightarrow v$ in $L^{2}$ norm as $\delta \rightarrow 0$. Hence we may apply (2.15) to $v_{\delta}$ to conclude that $\|v\|_{(\varepsilon, 1)}$ $<\infty$, and hence $\|v\|_{(\varepsilon, 2)}<\infty$ provided that we can show that $\left\|L v_{\delta}\right\| \|^{\prime}$ remains bounded as $\delta \rightarrow 0$. To prove the last assertion we prepare some remarks.

$1^{\circ}$. We have

$$
\begin{aligned}
& K_{1}(x)=\frac{1}{2} e^{-|x|}=(2 \pi)^{-1} \int_{-\infty}^{\infty} e^{i x \cdot \xi} \frac{d \xi}{1+\xi^{2}}, \quad-\infty<x<\infty, \\
& K_{2}(x)=C_{2}\left[1+\sqrt{|x|]^{-1}\left[1+\log \left(1+|x|^{-1}\right)\right] e^{-|x|}}\right. \\
&=(2 \pi)^{-2} \iint_{-\infty}^{\infty} e^{i\left(x_{1} \xi_{1}+x_{2} \xi_{2}\right)} \frac{d \xi_{1}+d \xi_{2}}{1+\xi_{1}^{2}+\xi_{2}^{2}}, \quad x \in R^{2} \backslash\{0\}, \\
& K_{\ell}(x)=C_{\ell}|x|^{2-\ell} e^{-|x|}=(2 \pi)^{-\ell} \int_{-\infty}^{\infty} \cdots \int e^{i\langle x, \xi\rangle} \frac{d \xi_{1} \cdots d \xi_{\ell}}{1+|\xi|^{2}}, \\
& \ell \geqq 3, \quad x \in \boldsymbol{R}^{\ell} \backslash\{0\} .
\end{aligned}
$$

$2^{\circ}$. If $Q$ is a differential operator of order $j \leqq 2$ in $\partial / \partial x$ and $\partial / \partial t$ with coefficients in $C^{\infty}(\bar{\Omega})$, it follows that

$$
\left\|\left(1-\delta^{2} \Delta\right)^{-1} Q u\right\| \leqq C\|u\|, \quad u \in L^{2}(\Omega) \cap \mathscr{E}^{\prime}(\Omega) .
$$

$3^{\circ}$. When $\chi \in C_{0}^{\infty}(\Omega)$ we have

$$
\left\|\chi \chi\left(1-\delta^{2} \Delta\right)^{-1} w\right\| \mid \leqq C\|w\| \|, \quad w \in C_{0}^{\infty}(\Omega) .
$$

Indeed we have for $w \in C_{0}^{\infty}(\Omega)$

$$
\begin{gathered}
\left|\sum_{k, j=1}^{m}\left\langle\mathscr{R}_{e} a^{k j} \cdot\left(\chi\left(1-\delta^{2} \Delta\right)^{-1} w\right)_{x_{h}},\left(\chi\left(1-\delta^{2} \Delta\right)^{-1} w\right)_{x_{j}}\right\rangle\right| \\
\leqq C\left(\|w\|^{2}+\left\|\chi \sqrt{\mathscr{R} e A} \cdot\left(1-\delta^{2} \Delta\right)^{-1} D_{x} w\right\|^{2}\right) .
\end{gathered}
$$

Here we denoted by $D_{x} w=\left(w_{x_{1}}, \cdots, w_{x_{m}}\right)$ and by $\|F\|^{2}=\left\|F_{1}\right\|^{2}+\cdots+$ $\left\|F_{m}\right\|^{2}$ for a $m$-vector $F=\left(F_{1}, \cdots, F_{m}\right)$. If we denote by $\mathfrak{U}=\chi \sqrt{\mathscr{R} e} A, \mathfrak{U}$ is an $m \times m$ matrix which is uniformly Lipschitz continuous and compactly supported in $\Omega$ by the results of [1]. Now for the second term of the right hand side, we have

$$
\begin{aligned}
\| \mathfrak{U}(1 & \left.-\delta^{2} \Delta\right)^{-1} D_{x} w \|^{2} \\
& =\left\|\left(1-\delta^{2} \Delta\right)^{-1} \mathfrak{U} D_{x} w+\left[\mathfrak{U} D_{x},\left(1-\delta^{2} \Delta\right)^{-1} I\right] w\right\|^{2}
\end{aligned}
$$




$$
\begin{aligned}
& \leqq 2\left\|\mathfrak{U} D_{x} w\right\|^{2}+2\left\|\left[\mathfrak{Y} D_{x},\left(1-\delta^{2} \Delta\right)^{-1} I\right] w\right\|^{2} \\
& \leqq\left. C\|w\|\right|^{2}+2\left\|\left[\mathfrak{U} D_{x},\left(1-\delta^{2} \Delta\right)^{-1} I\right] w\right\|^{2}
\end{aligned}
$$

Partial integration proves

$$
\begin{aligned}
& {\left[\mathfrak{U} D_{x},\left(1-\delta^{2} d\right)^{-1} I\right] w(x, y, t)} \\
& \begin{array}{l}
=\delta^{-(m+1)} \int_{R_{\xi, \tau}^{m+1}} K_{m+1}\left(\frac{x-\xi}{\delta}, \frac{t-\tau}{\delta}\right)[\mathfrak{U}(x, y, t) \\
\quad-\mathfrak{U}(\xi, y, \tau) D_{\xi} w(\xi, y, \tau) d \xi d \tau
\end{array} \\
& \begin{array}{r}
=\delta^{-(m+1)} \int_{R^{m+1}} K_{m+1}\left(\frac{x-\xi}{\delta}, \frac{t-\tau}{\delta}\right) w(\xi, y, \tau) D_{\xi} \mathfrak{U}(\xi, y, \tau) d \xi d \tau \\
=\delta^{-(m+2)} \int_{R^{m+1}} w(\xi, y, \tau) \cdot[\mathfrak{U}(x, y, t) \\
\quad-\mathfrak{U}(\xi, y, \tau)] D_{\xi} K_{m+1}\left(\frac{x-\xi}{\delta}, \frac{t-\tau}{\delta}\right) d \xi d \tau .
\end{array}
\end{aligned}
$$

By account of the explicit expression of $K_{m+1}$ in $1^{\circ}$ and by the uniform Lipschitz continuity of $\mathfrak{A}$, the $L^{2}$ norm of last two terms is bounded above by $\|w\|^{2}$. This estimate combined with (3.3) gives (3.2).

Completion of the proof of Lemma 3.1: We recall that it remains to prove that $\| L v_{\delta}||^{\prime}$ is bounded as $\delta \rightarrow 0$. In the neighbourhood $\omega$ of supp. $v$ we have $\left(1-\delta^{2} \Delta\right) v_{\delta}=v$ and

$$
\begin{aligned}
\left(1-\delta^{2} \Delta\right) L v_{\delta} & =L v-\delta^{2}[L, \Delta] v_{\delta} \\
& =L v+2 \delta^{2} \sum_{\sigma=1}^{m} \sum_{k, j=1}^{m}\left(a_{x_{\sigma}}^{k j} v_{\delta x_{k} x_{\sigma}}\right)_{x_{j}}+2 \delta^{2} \sum_{\dot{v}, j=1}^{m}\left(\alpha_{t}^{k j} v_{\delta x_{k} t}\right)_{x_{j}}+\delta^{2} B v_{\delta},
\end{aligned}
$$

where $B$ is a differential operator of the form

$$
B=\sum_{\substack{\alpha\left|+\alpha^{\prime} \leq 2 \\ \alpha\right| \leq 2 \leq 1 \\ \beta \mid \leq 2, \alpha^{\prime} \leqq 1}} B^{\alpha, \alpha^{\prime}, \beta}(x, y, t) D_{x}^{\alpha} D_{y}^{\beta} D_{t}^{\alpha^{\prime}},
$$

which may be considered with compact support. It follows that we have everywhere

$$
\begin{aligned}
\left(1-\delta^{2} \Delta\right) L v_{o}= & L v+2 \delta^{2} \sum_{\sigma=1}^{m} \sum_{k, j=1}^{m}\left(a_{x_{\sigma}}^{k j} v_{\delta x_{k} x_{\sigma}}\right)_{x_{j}}+2 \delta^{2} \sum_{k, j=1}^{m}\left(a_{t}^{k j} v_{\delta x_{k} t}\right)_{x_{j}} \\
& +\delta^{2} B v_{\sigma}+h_{\delta}
\end{aligned}
$$

where $h_{\delta}$ is a function such that it vanishes in $\omega$, supp. $h_{\delta} \subset \operatorname{supp} . \chi$ and $\left\|h_{\delta}\right\| \rightarrow 0$ as $\delta \rightarrow 0$ in view of $1^{\circ}$. Hence

$$
\begin{aligned}
L v_{\delta}=\chi_{1}\left\{\left(1-\delta^{2} \Delta\right)^{-1} L v\right. & +2 \delta^{2} B_{1} v_{\delta}+2 \delta^{2} B_{2} v_{\delta} \\
& \left.+\left(1-\delta^{2} \Delta\right)^{-1} \delta^{2} B v_{\delta}+\left(1-\delta^{2} \Delta\right)^{-1} h_{\delta}\right\},
\end{aligned}
$$


where $\chi_{1}$ is a function in $C_{0}^{\infty}(\Omega)$ which is equal to 1 in supp. $\chi$. We remark that from $3^{\circ}$ we have

$$
\left.\left\|\chi_{1}\left(1-\delta^{2} \Delta\right)^{-1} f\right\|\right|^{\prime} \leqq C\|\| f \|\left.\right|^{\prime}, \quad f \in \mathscr{D}^{\prime}(\Omega) \cap \mathscr{E}^{\prime}(\Omega) .
$$

Therefore, it follows that

$$
\left.\left\|\chi_{1}\left(1-\delta^{2} \Delta\right)^{-1} L v\right\|\right|^{\prime} \leqq\left. C\|L v\|\right|^{\prime} .
$$

The last two terms are bounded in $L^{2}$ norm in view of $2^{\circ}$ and by the assumption that $v \in H_{(0,2)}(\Omega) \cap \mathscr{E}^{\prime}(\Omega)$. For the second and third terms, we use (2.6), $2^{\circ}$ and (3.5) then we have

$$
\left.\left\|\delta^{2} B_{1} v_{\delta}\right\|\right|^{\prime}+\left.\left\|\delta^{2} B_{2} v_{\delta}\right\|\right|^{\prime} \leqq C\left\|v_{\delta}\right\| \leqq C^{\prime}\|v\| .
$$

Thus we have $\left.\left\|L v_{\delta}\right\|\right|^{\prime}<\infty$ as $\delta \rightarrow 0$. This completes the proof of Lemma 3.1 .

Proof of Theorem 1.1. The following process is almost the same as in $\S 3$ of [8].

Given a function $\psi \in C_{0}^{\infty}(\Omega)$ and an integer $r \geqq 2$, we may assume, by the partial hypoellipticity of $L$ in the direction $y$ (cf. [2], §4.3), that $\psi u \in H_{(s, 1)}\left(\boldsymbol{R}^{m+n+1}\right) \cap \mathscr{E}^{\prime}(\Omega)$ for some real number $s$. For the proof of Theorem 1.1 it suffices to show that $s$ can be replaced by $s+\varepsilon$. Indeed, it follows that $v \in H_{(s, r)}^{\mathrm{ioc}}(\Omega)$ for any $s$ and $r$, which means that $u \in C^{\infty}(\Omega)$ by the Sobolev lemma.

Let $E$ be a pseudo-differential operator with symbol $e(\xi, \tau)=\left(1+|\xi|^{2}\right.$ $\left.+|\tau|^{2}\right)^{s / 2}$ (cf. [4]), and set $v=\chi E \psi u$ where $\chi \in C_{0}^{\infty}(\Omega)$. If we can show that $v \in H_{(\varepsilon, r)}$ for every $\chi$ and $\psi$ we will have $E \psi u \in H_{(\varepsilon, 2)}^{\text {loc }}(\Omega)$, hence $u \in$ $H_{(s+\varepsilon, 2)}^{\text {loc }}$ since $E$ is elliptic. As $r \geqq 2$, it is clear that $v \in H_{(0,2)}(\Omega) \cap \mathscr{E}^{\prime}(\Omega)$, so in view of Lemma 3.1 it remains only to show that $\|L v\| \|^{\prime}<\infty$. We note that $E^{\prime}=\chi E \psi$ is considered as a compactly supported pseudodifferential operator of order $s$ (in $\left(x, x^{\prime}\right)$ ) with parameter $y$ (cf. [4]) and $L v=L E^{\prime} u$. Taking account of $E^{\prime} L u=E^{\prime} f$ and $L v=E^{\prime} f+L E^{\prime} u-E^{\prime} f$, it now suffices to show that $\left.\left\|L E^{\prime} u-E^{\prime} f\right\|\right|^{\prime}<\infty$ to prove $\|L v\| \|^{\prime}<\infty$. We have

$$
\begin{aligned}
L E^{\prime} u-E^{\prime} f= & \sum_{k, j=1}^{m}\left[a^{k j} D_{x_{k}} D_{x_{j}}, E^{\prime}\right] u-\sum_{k, j=1}^{m}\left[a_{x_{j}}^{k j} D_{x_{k}}, E^{\prime}\right] u \\
& +\sum_{j=1}^{n} E_{0}^{j} u_{y_{j}}+E_{1} u
\end{aligned}
$$


where $\sum_{k, j=1}^{m}\left[a_{x j}^{k j} D_{x_{k}}, E^{\prime}\right], E_{0}^{j}$ and $E_{1}$ are compactly supported pseudo-differential operators of order $\leqq s$ in the direction $(x, t)$ and we have

$$
\sum_{k, j=1}^{m}\left\|\left[\alpha_{x_{j}}^{k j} D_{x_{k}}, E^{\prime}\right] u\right\|+\sum_{j=1}^{n}\left\|E_{0}^{j} u_{y_{j}}\right\|+\left\|E_{1} u\right\|<0
$$

by assumption, so we have only to analyse the first summation in the right hand side. We have

$$
\begin{aligned}
{\left[\alpha^{k j} D_{x_{k}} D_{x_{j}}, E^{\prime}\right] u=} & {\left[a^{k j} D_{x_{k}}, E^{\prime}\right] D_{x_{j}} u+a^{k j} D_{x_{k}}\left[D_{x_{j}}, E^{\prime}\right] u } \\
= & D_{x_{j}}\left[a^{k j} D_{x_{k}}, E^{\prime}\right] u+\left[D_{x_{j}},\left[a^{k j} D_{x_{k}}, E^{\prime}\right]\right] u \\
& +a^{k j} D_{x_{k}}\left[D_{x_{j}}, E^{\prime}\right] u .
\end{aligned}
$$

On the other hand, if we denote by $\sigma(E)$ a symbol of a pseudo-differential operator $E$, a simple calculation (cf. [4]) proves the equality

$$
\sigma\left(\left[a^{k j} D_{x_{k}}, E^{\prime}\right]\right)=a^{k j} \sigma\left(E_{1}^{k}\right)+\sum_{\nu=1}^{m} a_{x_{\nu}}^{k j} \sigma\left(E_{2}^{\nu}\right)+a_{t}^{k j} \sigma\left(E_{3}\right)+\sigma\left(E_{4}\right)
$$

where $E_{1}^{k}(k=1, \cdots, m), E_{2}^{\nu}(\nu=1, \cdots, m), E_{3}$ and $E_{4}$ are pseudo-differential operators with parameter $y$ of order $\leqq s$ and $\leqq s-1$ respectively (in the direction $(x, t)$ ). This equality leads us, by using (2.6), $\left(2.6^{\prime}\right)$ and (2.7)

$$
\left\|\sum_{k, j=1}^{m} D_{x_{j}}\left[a^{k j} D_{x_{k}}, E^{\prime}\right] u\right\| \|^{\prime}<\infty .
$$

Obviously we have

$$
\left\|\left[D_{x_{j}},\left[a^{k j} D_{x_{k}}, E^{\prime}\right]\right] u\right\| \|^{\prime}<\infty
$$

since the order of $\left[D_{x_{j}},\left[a^{k j} D_{x_{k}}, E^{\prime}\right]\right]$ is less than or equal to $s$. Finally for any $w \in C_{0}^{\infty}(\Omega)$ we have

$$
\begin{aligned}
\mid \sum_{k, j=1}^{m} & \left\langle a^{k j} D_{x_{k}}\left[D_{x_{j}}, E^{\prime}\right] u, w\right\rangle \mid \\
& =\left|\sum_{j=1}^{m}\left\langle\left[D_{x_{j}}, E^{\prime}\right] u, \sum_{k=1}^{m} a^{k j} w_{x_{k}}\right\rangle\right| \\
& \leqq C|||w| \|,
\end{aligned}
$$

hence we have

$$
\left\|\sum_{k, j=1}^{m} a^{k j} D_{x_{k}}\left[D_{x_{j}}, E^{\prime}\right] u\right\| \|^{\prime}<\infty .
$$

The above investigation implies that 


$$
\left.\left\|L E^{\prime} u-E^{\prime} f\right\|\right|^{\prime}<\infty .
$$

Thus we have $\|L v\| \|^{\prime}<\infty$ and this completes the proof of Theorem 1.1.

\section{REFERENCES}

[1] Freidlin, M. I.: On the factorization of nonnegative definite matrices, Theor. Probability Appl., 13 (1968), 354-356.

[ 2 ]Hörmander, L.: Linear partial differential operators, Springer Verlag, 1964.

[ 3 ]Hörmander, L.: Hypoelliptic second order differential equations, Acta Math., 119 (1968), 147-171.

[4] Hörmander, L.: Fourier integral operators, I, Acta Math., 127 (1971), 79-183.

[5] Kato, Y.: On a class of hypoelliptic differential operators, Proc. Japan Acad., 46, No. 1 (1970), 33-37.

[6] Kohn, J. J.: Pseudo-differential operators and hypoellipticity, Berkeley Symposium (1972).

[ 7] Matsuzawa, T.: On some degenerate parabolic equations I, Nagoya Math. J. 51 (1973), 57-77, II, Nagoya Math. J. 52 (1973), 61-84.

[8] Matsuzawa, T.: On a hypoelliptic boundary value problem, Nagoya Math. J. (to appear).

[9] Oleinkik, O. A. and Radkevič, E. V.: Second order equations with nonnegative characteristic form, Amer. Math. Soc., 1973.

Department of Mathematics,

Nagoya University 